\title{
Design of Plasmonic Slot Waveguide with High Localization and Long Propagation Length
}

\author{
Ki-Sik Lee and Jaehoon Jung* \\ Department of Electronics and Electrical Engineering, Dankook University, Yongin 448-701, Korea
}

(Received August 30, 2011 : revised August 31, 2011 : accepted September 5, 2011)

\begin{abstract}
We present an efficient design approach for a plasmonic slot waveguide using a genetic algorithm. The analyzed structure consists of a nanometric slot in a thin metallic film embedded within a dielectric. To achieve high confinement without long propagation length, the thickness and width of the slot are optimally designed in order to optimize the figures of merit including mode confinement and propagation length. The optimized design is based on the finite element method and enhances the guiding and focusing of light power propagation.
\end{abstract}

Keywords : Surface plasmon polariton, Waveguide, Genetic algorith

OCIS codes : (240.6680) Surface plasmons; (130.2790) Guided waves; (230.7370) Waveguides

\section{INTRODUCTION}

Plasmonic waveguides have attracted great attention for deep subwavelength confinement beyond the diffraction limit compared to conventional dielectric-based ones [1, 2]. The rising interest in such waveguides lies in the fact that their propagation modes have decay lengths that are perpendicular to the propagation direction and are much smaller than the excitation wavelength. They are considered as alternatives applicable to highly integrated photonic circuits such as filters [3], modulators [4], sensors [5, 6], optical attenuators [7] and switches [8]. However, highly confined modes involve enhanced propagation losses that are typically due to Ohmic loss in the metal and therefore there is a fundamental trade-off between light confinement and propagation loss.

In recent years, surface plasmon polaritons (SPPs) in the metal slot have been thoroughly investigated and reported to feature a well-balanced tradeoff between localization and propagation loss [9-16].

In this paper, we investigate the fundamental waveguide parameters of slot waveguides at the telecom wavelength $(1550 \mathrm{~nm})$ such as an effective refractive index, propagation length and figure of merit (FOM) including both the confinement and loss effects previously reported [15]. First, we investigate the effects of variation in slot thickness and width on mode confinement and propagation length. Furthermore, an efficient design approach is provided to obtain a slot wave- guide with optimal performance parameters. We will optimize the waveguide geometry by using the genetic algorithm (GA). Through the analysis and design procedure, we identify the intrinsic tradeoffs and suggest an optimal geometry of a plasmonic waveguide with considerable propagation length and deep-subwavelength localization with strong field enhancement. The field analysis will be carried out through the finite element method (FEM).

\section{ANALYSIS OF PLASMONIC SLOT WAVEGUIDES}

The geometry of a plasmonic slot waveguide is shown in the inset of Fig. 1 (a). It consists of a slot in a thin metallic film with width $w$ and thickness $h$ embedded within a dielectric. It is assumed that the wave propagates in the $+z$-direction. In this paper, the metal is silver, the dielectric silica, and the excitation wavelength is $1550 \mathrm{~nm}$ unless otherwise specified. The dielectric constant of silver is $-86.6424+8.7422 \mathrm{i}$ at $1550 \mathrm{~nm}$ [17]. The electric field of the propagation mode supported by the plasmonic slot waveguide can be written as $\vec{E}=\vec{E}_{0}(x, y) \exp [i(\omega t-\beta z)]$. The mode effective index is defined as $n_{\text {eff }}=\operatorname{real}(\beta) / k_{0}$ and the propagation length as $L_{s p}=1 / \operatorname{imag}(\beta)$ where $k_{0}$ is a propagation constant in a vacuum. The characteristics of

\footnotetext{
*Corresponding author: andyjung@dku.edu

Color versions of one or more of the figures in this paper are available online.
} 
propagation modes have been investigated in detail using the FEM through the commercially available COMSOL software package. The propagation power of each mode is normalized and can be written as $\frac{1}{2} \iint(\vec{E} \times \vec{H}) \cdot \hat{z} d a=1$.

Fig. 1(a) shows the vertical field distribution of the dominant electric-field component along the center of the slot with $w=100 \mathrm{~nm}$ and $h=100 \mathrm{~nm}$. The dimensions of the slot waveguide are much smaller than the excitation wavelength, whereas the field distribution can be focused around the slot due to the charge distribution in the metal surface. The electric field in this waveguide has a dominant component in the $x$ direction, since there are more rapid changes in the slot laterally than vertically.

In Fig. 1(b), we show the lateral distribution of the dominant electric-field component along the center of the slot with $w=100 \mathrm{~nm}$ and $h=100 \mathrm{~nm}$. It is clear that the electric field reaches the maximum at the interfaces between the metal and dielectric. The field distribution is also symmetrical with respect to the center of the slot, implying an antisymmetric charge distribution in the metal.

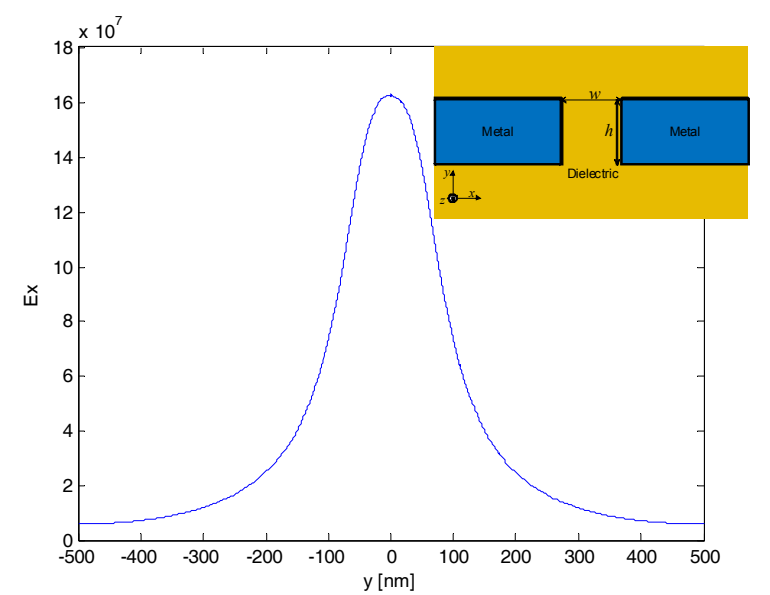

(a)

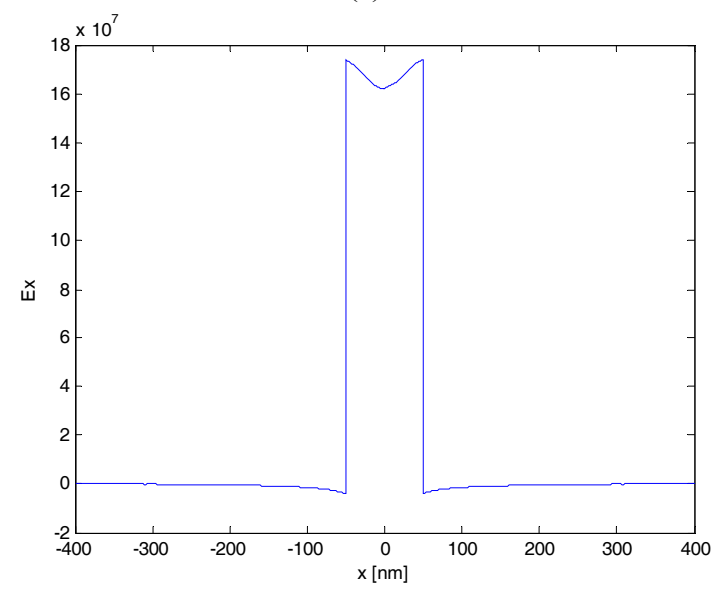

FIG. 1. (a) The vertical field distribution of the dominant electric-field component and (b) the lateral distribution of the dominant electric-field component along the center of the slot with $w=100 \mathrm{~nm}$ and $h=100 \mathrm{~nm}$.
We first investigate the effect of the slot dimension on the propagation characteristics. In Fig. 2 (a) we show the effective index $n_{\text {eff }}$ and propagation length $L_{s p}$ of the propagation mode of the slot plasmonic waveguide as a function of the slot width $w$ for different slot thicknesses. As the slot width $w$ decreases, the electric field in the slot increases and the electron mobility in the propagation direction decreases, thus resulting in a slower phase velocity and higher effective index of the propagation mode of the waveguide. The propagation length increases monotonically as the slot width increases. It is also important to note that decreasing the thickness of the slot $h$ results in a larger electric field in the slot, leading to high $n_{\text {eff }}$ and low $L_{s p}$. These results are consistent with a trade-off between mode localization and propagation length, that is, the higher the confinement, the shorter the propagation length. Thus, we use an FOM as an evaluation metric of waveguide performance, $L_{s p} \sqrt{\pi / A}$, which is similar to the proposal in [15] where $A$ is the bounded area defined by the closed 1/e field magnitude relative to the field at the center of the slot.

In Fig. 2 (b) we show the FOM of the propagation mode

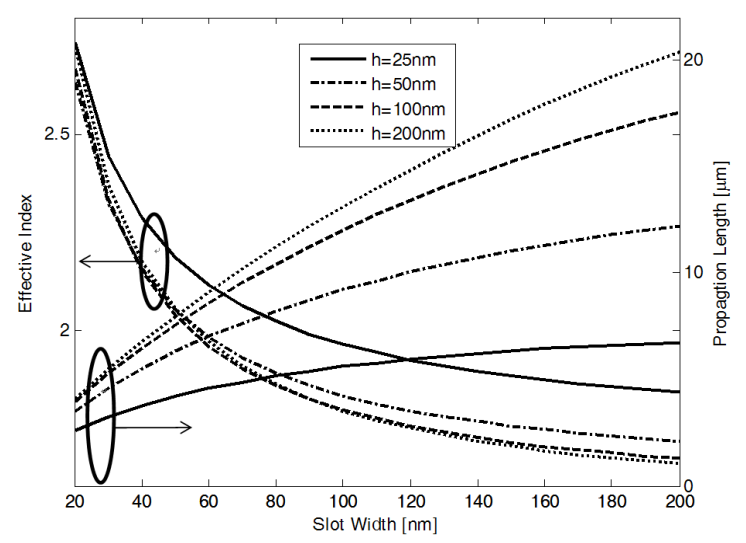

(a)

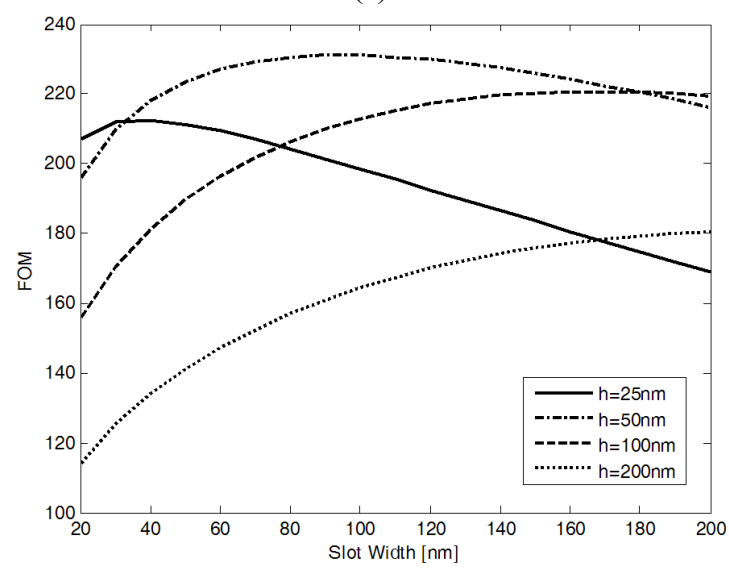

(b)

FIG. 2. (a) The effective index and propagation length of the propagation mode of the slot plasmonic waveguide and (b) FOM of the propagation mode of the slot plasmonic waveguide as a function of the slot width $w$ for different slot thicknesses. 
of the slot plasmonic waveguide as a function of the slot width $\mathrm{w}$ for different slot thicknesses. Unlike $n_{\text {eff }}$ and low $L_{s p}$, the FOM does not exhibit monotonic behavior, because as the slot width increases, the propagation length increases but the mode size also increases according to the ratio, resulting in a maximum.

In Fig. 3 (a) we show $n_{\text {eff }}$ and $L_{s p}$ of the fundamental mode of the slot waveguide as a function of the slot thickness $h$ according to the slot width. It is clear that as the slot width increases, $n_{\text {eff }}$ decreases and $L_{s p}$ increases. However, the dependence of the slot thickness on $n_{\text {eff }}$ is less obvious, since decreasing the slot thickness causes the field to extend from the slot into the cladding.

In Fig. 3 (b) we show the FOM of the fundamental mode of the slot waveguide as a function of slot thickness according to slot width. It is clear that the FOMs are not monotonic, but reach a maximum around $30-100 \mathrm{~nm}$, and this is therefore a local maximum. In these cases, an optimization approach based on gradient, e.g. sequential quadratic programming (SQP), would be more likely to obtain a local maximum and depend on its initial value.

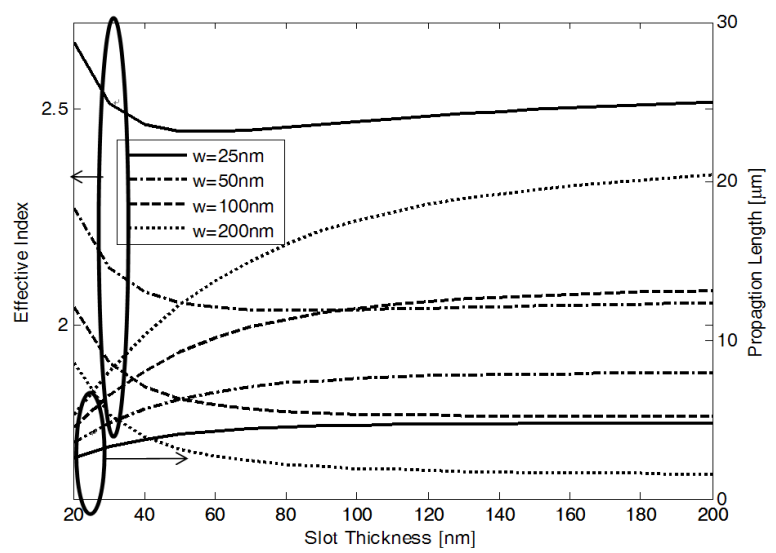

(a)

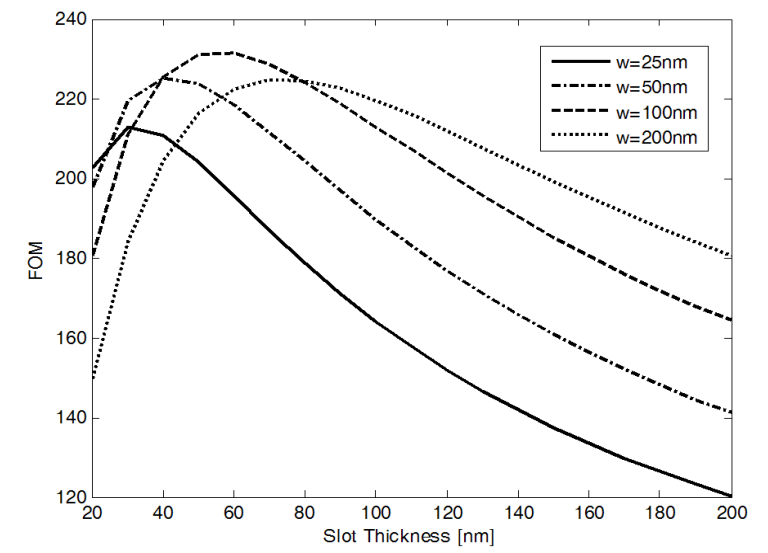

(b)

FIG. 3. (a) The effective index and propagation length of the propagation mode of the slot plasmonic waveguide and (b) FOM of the propagation mode of the slot plasmonic waveguide as a function of the slot thickness $h$ for different slot widths.
In Fig. 4 (a), (b), and (c) we show $n_{e f f}, L_{s p}$, and FOM of the propagation mode of the slot waveguide in different geometries having the same slot area. The expressions in the parenthesis represent the width and thickness, respectively. It is clear that the slot width has a dominant effect on the effective index, indicating that the phase velocity changes abruptly according to slot width. It is noteworthy that the propagation length trend is inversely related to that of the effective index.

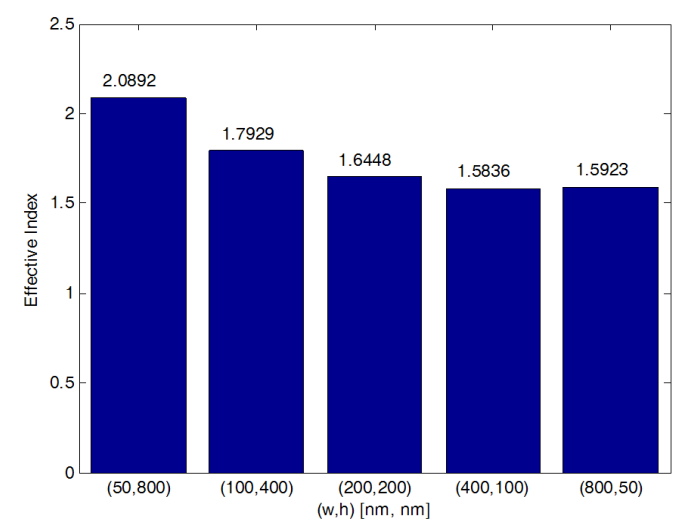

(a)

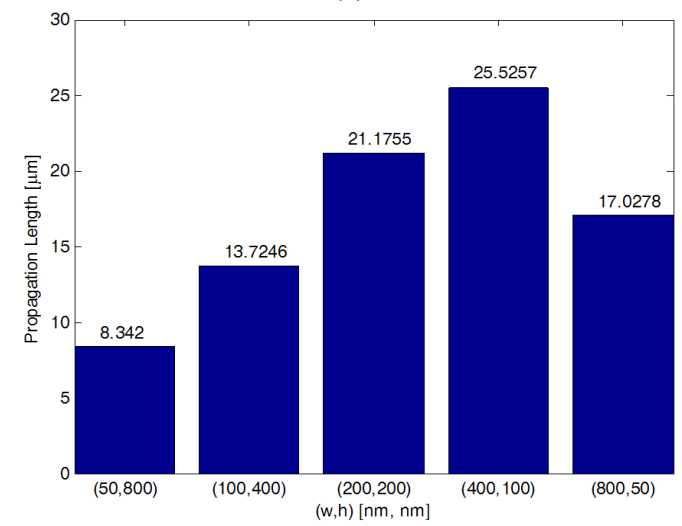

(b)

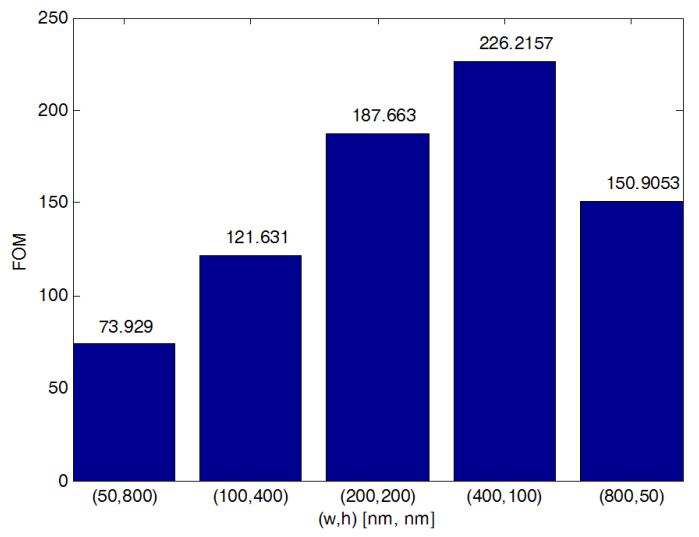

(c)

FIG. 4. (a) The effective index, (b) propagation length of the propagation mode of the slot plasmonic waveguide and (c) FOM of the propagation mode of the slot waveguide in different geometries having the same slot area. 


\section{OPTIMAL DESIGN USING GENETIC ALGORITHM}

The GA approach is applied to the optimization of the plasmonic slot waveguide in order to design waveguide parameters. The GA is a powerful and efficient method for optimization problems of a given function with many design parameters. It is based on a stochastic technique, and a population of individuals is randomly created at each generation. In our computations, the objective function is FOM, as previously defined, and the design parameters are the width $w$ and thickness $h$ of the slot where the electric field is concentrated. The GA parameters are set as follows: the population size was 100 , the crossover rate was 0.8 , and hence the mutation rate was 0.2 . The number of generations was fixed at 100 runs. The lower and upper search bounds of both design parameters are $20 \mathrm{~nm}$ and $400 \mathrm{~nm}$, respectively. The computational optimization result shows that the optimal parameters of the slot were $w=180.3 \mathrm{~nm}$ and $h=$ $68.3 \mathrm{~nm}$ and its corresponding FOM is 237.4396 for optimization generation. The optimum design results and the case comparisons are summarized in Table 1. Case III corresponds to the geometry having the largest FOM in Fig. 3 (b).

Fig. 5 shows the electric field distribution of the plasmonic slot waveguides for the different cases in Table 1. The numerical results show that the mode profile is confined around the corners of the slot. Their field distributions remain fundamentally unchanged with the exception of a slight change in mode confinement and corresponding propagation length, whereas there are considerable changes in FOM values.

In Fig. 6 (a) and (b), we show the vertical and lateral distribution of the dominant electric-field component along the center of the slot for the different cases in Table 1, respectively. Comparing the optimal case with cases I-III, it is clear that in the optimal case, the mode confinement is higher than for case III, but its FOM is the maximum, because in case III, the propagation length is much shorter.

TABLE 1. Optimum parameters of the GA

\begin{tabular}{c|c|c|c|c}
\hline \hline & Case I & Case II & Case III & Optimal \\
\hline$w(\mathrm{~nm})$ & 100 & 200 & 100 & 180.3 \\
\hline$h(\mathrm{~nm})$ & 100 & 200 & 60 & 68.3 \\
\hline FOM & 213.8 & 187.7 & 232.7 & 237.4 \\
\hline
\end{tabular}

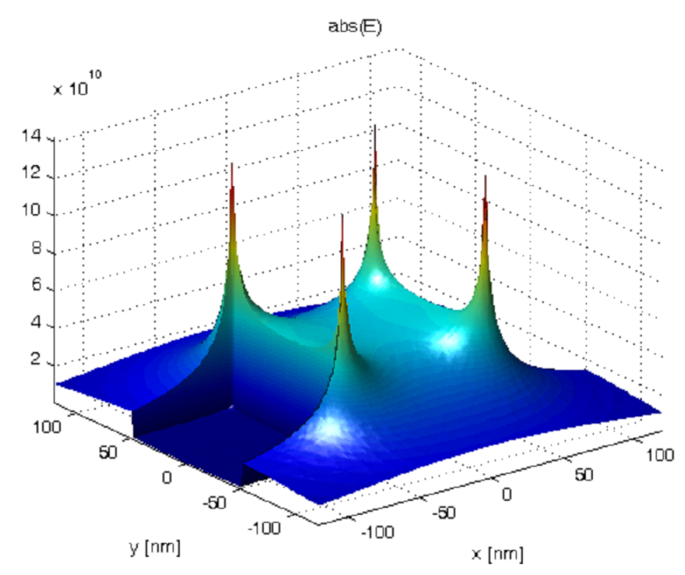

(a)

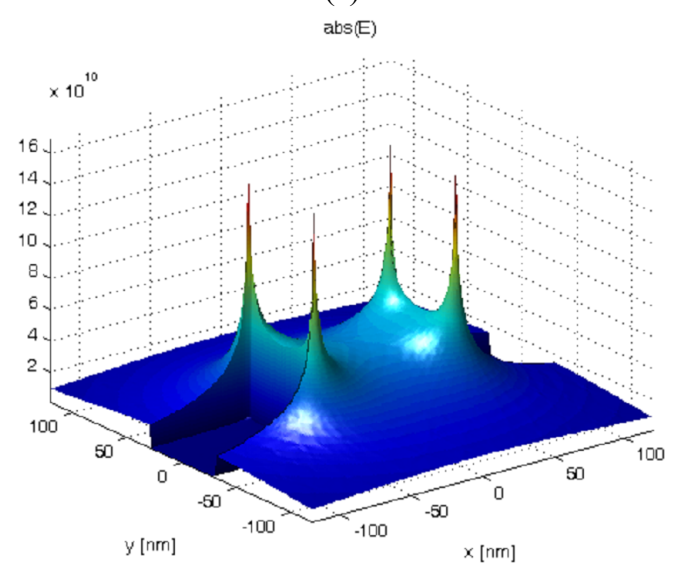

(c)

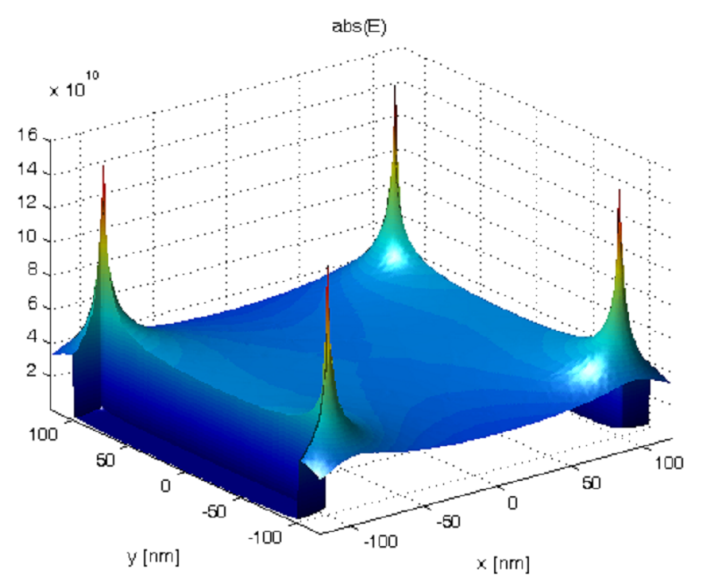

(b)

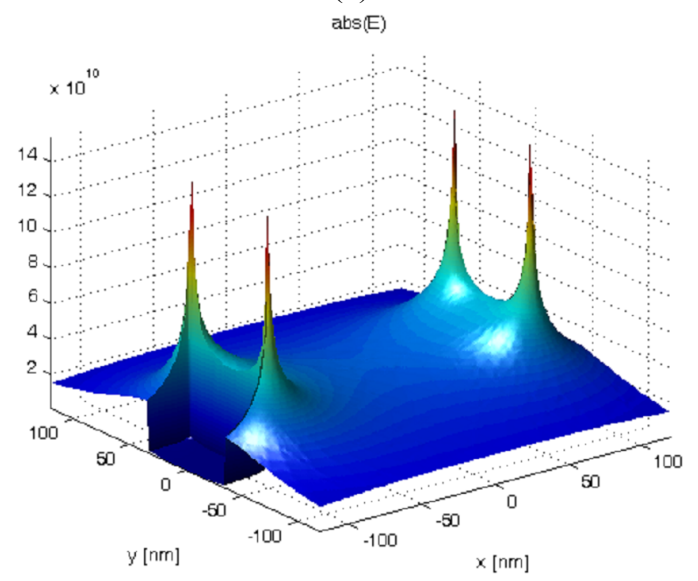

(d)

FIG. 5. The electric field distribution for the plasmonic slot waveguides of the cases in Table 1: (a) Case I, (b) Case II, (c) Case III, (d) Optimal Case. 


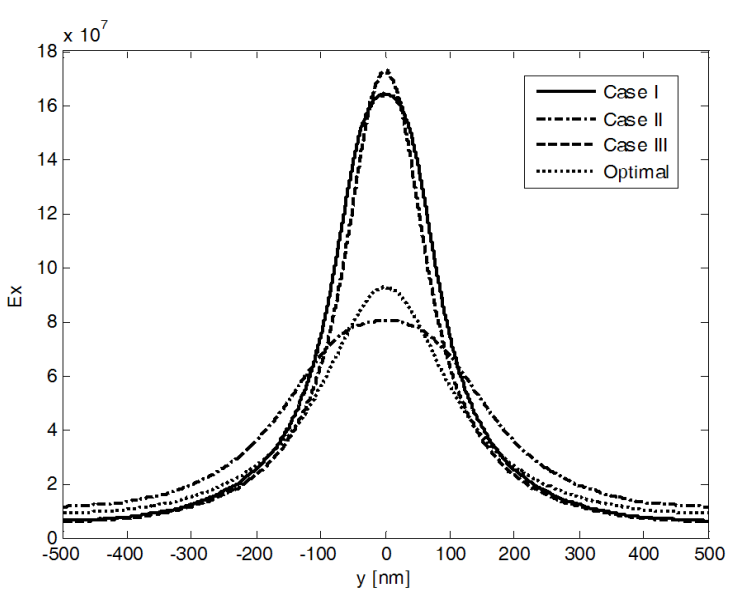

(a)

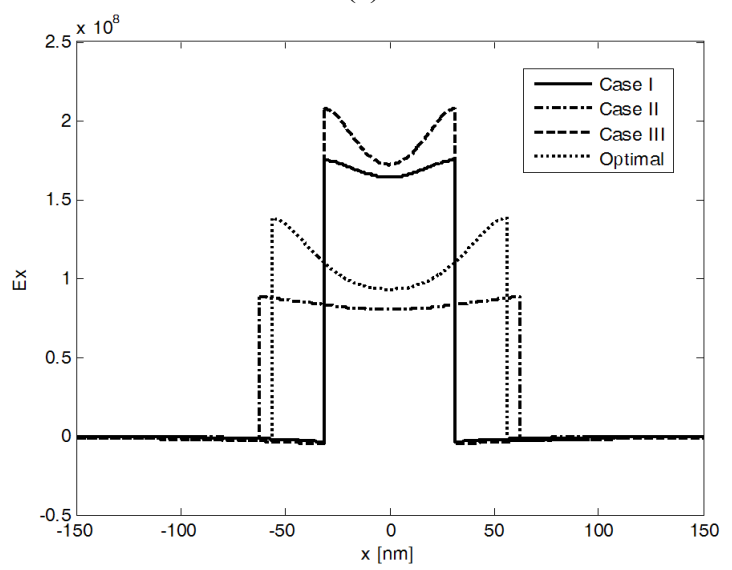

(b)

FIG. 6. The vertical (a) and lateral (b) distribution of the dominant electric-field component along the center of slot for different cases.

\section{CONCLUSION}

A numerical study of propagation characteristics of the plasmonic slot waveguide has been performed. We used the FEM and investigated the effect of the slot dimension on propagation characteristics such as the effective refractive index, propagation length and FOM including both confinement and loss effect. The field distributions of the propagation mode for the slot waveguide were investigated numerically in detail. Furthermore, using GA, we presented an efficient and powerful approach to the optimization of the plasmonic slot waveguide for high mode confinement and long propagation length, between which there is an intrinsic tradeoff. The approach proposed here has important implications for designing optimal plasmonic waveguides applicable to highly integrated nanometric optical circuits.

\section{ACKNOWLEDGMENT}

This work was supported by the Dankook University project for funding Research Institute of Information and Communications Convergence Technology.

\section{REFERENCES}

1. H. Raether, Surface Plasmons on Smooth and Rough Surfaces and Gratings (Springer-Verlag, Berlin, Germany, 1988).

2. J. Takahara, S. Yamagishi, H. Taki, A. Morimoto, and T. Kobayashi, "Guiding of a one-dimensional optical beam with nanometer diameter," Opt. Lett. 22, 475-477 (1997).

3. A. Boltasseva, S. I. Bozhevolnyi, T. Nikolajsen, and K. Leosson, "Compact Bragg gratings for long-range surface plasmon polaritons," J. Lightwave Technol. 24, $912-918$ (2006).

4. T. Nikolajsen, K. Leosson, and S. I. Bozhevolnyi, "In-line extinction modulator based on long-range surface plasmon polaritons," Opt. Comm. 244, 455-459 (2005).

5. D. Kim, "Effect of the azimuthal orientation on the performance of grating-coupled surface-plasmon resonance biosensors," Appl. Opt. 44, 3218-3223 (2005).

6. K.-M. Byun, "Development of nanostructured plasmonic substrates for enhanced optical biosensing," J. Opt. Soc. Korea 14, 65-76 (2010).

7. G. Gagnon, N. Lahoud, G. A. Mattiussi, and P. Berini, "Thermally activated variable attenuation of long-range surface plasmon-polariton waves," J. Lightwave Technol. 24, 4391-4401 (2006).

8. T. Nikolajsen, K. Leosson, and S. I. Bozhevolnyi, "Surface plasmon polariton based modulators and switches operating at telecom wavelengths," Appl. Phys. Lett. 85, 5833-5835 (2004).

9. G. Veronis and S. Fan, "Guided subwavelength plasmonic mode supported by a slot in a thin metal film," Opt. Lett. 30, 3359-3361 (2005).

10. L. Liu, Z. Han, and S. He, "Novel surface plasmon waveguide for high integration," Opt. Express 13, 6645-6650 (2005).

11. D. F. P. Pile, T. Ogawa, D. K. Gramotnev, Y. Matsuzaki, K. C. Vernon, K. Yamaguchi, T. Okamoto, M. Haraguchi, and M. Fukui, "Two dimensionally localized modes of a nanoscale gap plasmon waveguide," Appl. Phys. Lett. 87, 261114 (2005).

12. J. A. Dionne, H. J. Lezec, and H. A. Atwater, "Highly confined photon transport in subwavelength metallic slot waveguides," Nano Lett. 6, 1928-1932 (2006).

13. G. Veronis and S. Fan, "Modes of subwavelength plasmonic slot waveguides," J. Lightwave Technol. 25, 2511-2521 (2007).

14. N.-N. Feng, M. L. Brongersma, and L. D. Negro, "Metaldielectric slot-waveguide structures for the propagation of surface plasmon polaritons at $1.55 \mu \mathrm{m}$," IEEE J. Quantum Electron. 43, 479-485 (2007).

15. R. Buckley and P. Berini, "Figures of merit for 2D surface plasmon waveguides and application to metal stripes," Opt. Express 15, 12174-12182 (2007).

16. J. Jung, "Optimal design of dielectric-loaded surface plasmon polariton waveguide with genetic algorithm," J. Opt. Soc. Korea 14, 277-281 (2010).

17. E. D. Palik, Handbook of Optical Constants of Solids (Academic, New York, USA, 1985). 